CORRESPONDENCE

\section{Corneal transplantation and infectious hepatitis}

EDITOR,-I enjoyed reading the insightful review by Badenoch on the risks of transmission of the hepatitis virus in corneal transplantation. ${ }^{1}$ Among his conclusions, he pointed out that in geographical areas where there is a high prevalence of hepatitis B virus (HBV) infection and a scarcity of corneal graft material, an eye bank might be tempted to offer corneas from hepatitis B surface antigen (HBsAg) positive donors to $\mathrm{HBV}$ antibody (anti-HBs) positive recipients. $\mathrm{He}$ recommended that these tissues should only be used in an absolute emergency because of the as yet inadequate understanding of the transmission and pathogenesis of $\mathrm{HBV}$ and hepatitis D virus (HDV) infection.

In Hong Kong, as well as in some other places, the risk of transmitting $\mathrm{HBV}$ from $\mathrm{HBsAg}$ positive donors to $\mathrm{HBsAg}$ or anti-HBs positive recipients is considered to be so small that kidneys from HBsAg positive donors are frequently used for transplantation. ${ }^{23}$ Good long term results have been reported in HBsAg negative patients who received kidneys from $\mathrm{HBsAg}$ positive donors. ${ }^{3}$

Furthermore, the chance of viral transmission in transplantation of the avascular corneal tissues is much lower than that of the vascular organs. For example, AIDS is readily transmitted in the transplantation of vascular organs such as liver, kidney, and heart but no cases of transmission of the disease via corneal transplantation have been reported. ${ }^{4}$

The recommendation from Badenach must be treated with caution in places like Hong Kong, Taiwan, the southern part of China, and the Philippines where there is a high prevalence of $\mathrm{HBsAg}$ carriers (about $10-15 \%)^{5}$ and a low cornea donation rate Implementation of such a policy would further exaggerate the delay of corneal transplantation in blind patients. The chance of HBV infection through corneal transplantation is so small that the risk is probably outweighed by the prolonged suffering endured by patients with poor vision and poor quality of life.

For patients who have not been exposed to $\mathrm{HBV}$, transplantation of corneas from HBV positive donors is best avoided. Nevertheless, vaccination and seroconversion of these patients to anti-HBs positive status may allow them to take the option of receiving HBsAg positive donor tissues. In patients who are already anti-HBs positive, a booster dose of $\mathrm{HBV}$ vaccine to enhance their immune response, just before the corneal transplantation, may further minimise the chance of getting $\mathrm{HBV}$ infection.

In geographic areas where the corneal tissues are abundant, I agree that corneas from donors positive for HBV should not be used for transplantation. However, before the results of a large and well designed study to evaluate the actual risks of using corneal tissues from $\mathrm{HBsAg}$ positive donors to HBsAg or anti-HBs positive recipients are available, it is premature to recommend that these corneal tissues should be routinely discarded in all centres.
I would like to thank Johnson Y N Lau, MD, Section of Hepatobiliary Diseases, University of Florida, for his helpful discussion and encouragement.

DENNIS S C LAM

Department of Ophthalmology and Visual Sciences, The Chinese University of Hong Kong, Prince of Wales Hospital, Shatin, Hong Kong

1 Badenoch PR. Corneal transplantation and infectious hepatitis. $\mathrm{Br} f$ Ophthalmol 1995; 79: 2.

2 Bedrossian J, Akposso K, Metivier F, Moal MC, Pruna A, Idatte JM. Kidney transplantation with HBSAg + donors. Transplant Proc 1993; 25: 1481-2.

3 Cheng IK. Special issues related to transplantation in Hong Kong. Transplant Proc 1992; 24: 2423-5.

4 Simons RJ. HIV transmission by organ and tissue transplantation. AIDS 1993; 7: S35-8.

5 Yeoh EK. Hepititis B: the Asian threats. In: Douglas RG, ed. Assessment and management of risks associated with hyperlipidemia, osteoporosis and hepatitis B: effectiveness of intervention. Philadelphia: Jr Hanely \& Belfus Inc, 1990: 209-23.

\section{Orbital lymphoma in systemic} sarcoidosis

EDITOR,-Sarcoidosis is a systemic granulomatous disease of unknown origin, which may affect the lacrimal gland. The onset of a bilateral lacrimal gland enlargement in a patient with histology proved sarcoidosis is, therefore, generally considered as a sign of orbital involvement.

However, sarcoidosis is also characterised by a higher incidence of lymphoma ('sarcoidosis lymphoma syndrome'), 1 and the omission of biopsy can considerably delay the recognition of an associated lymphoma.

In September 1992, an 82-year-old woman was referred for the evaluation of painless proptosis, with bilateral lacrimal gland enlargement, which had arisen in 1987, with no inflammatory signs (Fig 1). As the patient had suffered since 1977 from biopsy proved pulmonary sarcoidosis, the orbital masses had been ascribed presumptively to sarcoidosis. Computed tomography scan revealed in both orbits well defined masses, moulding to the orbital walls, to the optic nerve, and to extraocular muscles, involving both lacrimal glands and enhancing after contrast administration (Fig 2). Left orbital biopsy revealed a small cell diffuse lymphocytic lymphoma (low grade). Systemic staging was negative. Orbital radiotherapy $(3500 \mathrm{cGy})$ obtained complete remission at last follow up (April 1995)

The present report is the first regarding an orbital lymphoma complicating sarcoidosis. The relation between these two affections

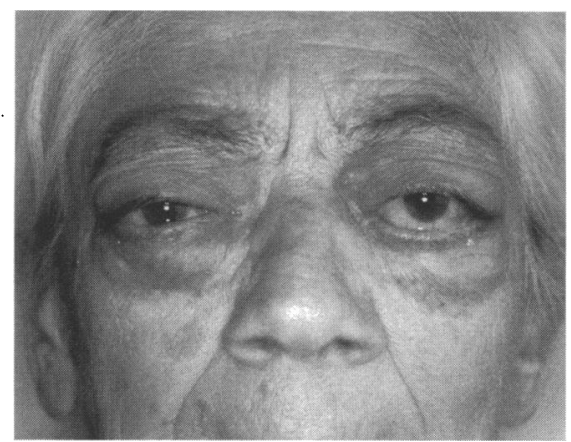

Figure 1 Clinical photograph at presentation. Axial proptosis, superotemporal and inferior masses are apparent in both orbits.

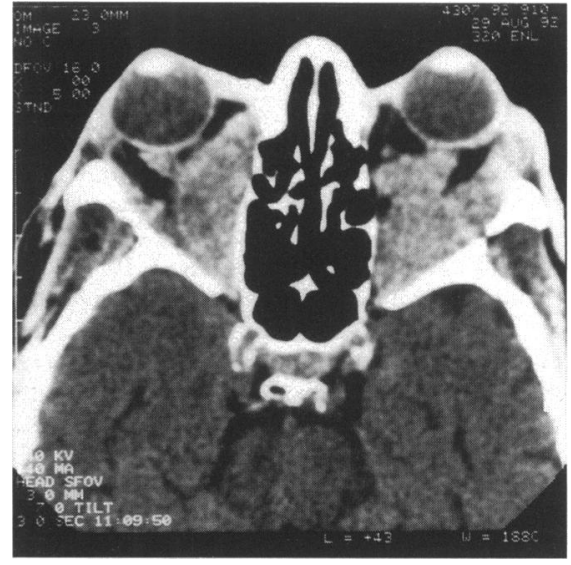

Figure 2 Axial computed tomogram reveals well delimited masses in both orbits, moulding to the orbital walls, to the optic nerve and extraocular muscles, and involving lacrimal glands.

is unclear. Both diseases are characterised by immunological abnormalities and, in sarcoidosis, an increased activity and a polyclonal growth of $\mathrm{T}$ cells is demonstrated. ${ }^{2}$ Although orbital lymphomas are generally constituted by monoclonal B cell proliferations, orbital reactive lymphoid hyperplasia is, in fact, a polyclonal lesion characterised by a majority of $\mathrm{T}$ cells $(60 \%)$, which may finally evolve into a B-cell lymphoma. ${ }^{3}$

As the present case shows, lymphoma may mimic lacrimal gland sarcoidosis and biopsy is needed for differential diagnosis. The computed tomogram finding of extralacrimal masses is very uncommon in sarcoidosis, and represents a stronger indication for biopsy.

ENNIO POLITO ANTONIO LECC Orbital and Adnexal Service,

Department of Ophthalmology and Neurosurgery University of Siena, Italy

1 Brincker $H$. The sarcoidosis-lymphoma syndrome. Br $\mathcal{F}$ Cancer 1986; 54: 467-73.

2 Lawrence E, Brousseau KP, Berger MB, Kurman CC, Marcon L, Nelson DL. Elevated concentration of soluble interleukin-2 receptors in serum samples and bronchoalveolar lavage fluid in active sarcoidosis. Am Respir Dis 1988; 137: 759-64.

3 Jakobiec FA, Neri A, Knowles DM II. Genotypic monoclonality in immunophenotypically polyclonal orbital lymphoid tumors. A model of tumor progression in the lymphoid system. Ophthalmology 1987; 94: 980-94.

Reducing bias during intraocular pressure measurement

EDITOR,-Intraocular pressure (IOP) measurement may be subject to a previously undescribed source of measurement bias. A simple modification of the Haag-Streit 900 slit-lamp will remove this source of error.

The existence of bias during IOP measurement has been documented already. Digit preference, especially for even numbers, was demonstrated for Goldmann applanation tonometry by Hollows and Graham. ${ }^{1}$ They also described the decision effect - that is, when an observer has to choose between normal and abnormal leading to a relative deficiency of $21 \mathrm{~mm} \mathrm{Hg}$ readings. We suggest a simple method to reduce one source of measurement bias.

When using the Goldmann tonometer, measurement bias can occur not just at the 